\title{
A Comparative Study between the K-Nearest Neighbors and the Multi-Layer Perceptron for Cursive Handwritten Arabic Numerals Recognition
}

\author{
B. El Kessab \\ Laboratory of Information \\ Processing and Decision Aids, \\ Faculty of Science and Technology \\ BP 523, Beni Mellal, Morocco
}

\author{
C. Daoui \\ Laboratory of Information \\ Processing and Decision Aids \\ Faculty of Science and Technology \\ BP 523, Beni Mellal, Morocco

\section{R. Salouan} \\ Department of Mathematic and \\ Informatic, Polydisciplinary Faculty \\ Sultan Moulay Slimane University, \\ Beni Mellal, MOROCCO
}

\author{
B. Boukhalene \\ Team Information Processing \\ Polydisciplinary Faculty \\ Sultan Moulay Slimane University, \\ Beni Mellal, MOROCCO
}

\begin{abstract}
In this paper we present a comparison between two supervised classifiers, the first one is a statistic which is the K-Nearest Neighbors (KNN) while the second is a neuronal which is the multi-layer perceptron MLP in the recognition of cursive handwritten Arabic numerals. The recognition process is organized as follows: in the pre-processing of numeral images, we exploited the median filter, the thresholding, the centering and the normalization techniques, in the features extraction we have used the morphology mathematical method. The classification methods include the KNN and the MLP. The simulation results that we obtained demonstrate the MLP is more efficient than the KNN in this recognition.
\end{abstract}

\section{General Terms}

The K-Nearest Neighbors (KNN), The multi-layer perceptron (MLP), The mathematical morphology method.

\section{Keywords}

The cursive handwritten Arabic numerals: The median filter, the thresholding, the centering and the normalization, techniques, the mathematical morphology method, the $\mathrm{K}$ Nearest Neighbors (KNN), The multi-layer perceptron (MLP).

\section{INTRODUCTION}

Optical character recognition systems has been widely developed in recent years, it can contribute to the advancement of office automation and can improve humancomputer interaction. On the hand, Handwritten recognition is one of the successful subcategories of optical character recognition that plays an important role in has many applications including archiving documents and automatic verification of bank checks, etc.
Since, several methods for recognition of handwritten Eastern Arabic, Romain, and Arabic numerals, have been proposed and realized, but the progress in cursive Arabic numerals recognition has been slow mainly due to many encountered difficulties in this recognition in reason of this last is carried in a manner on line that is to say a unknown numeral is recognized just after having writted him in a graphical interface of a certain software exploited in Optical character recognition. In this context, this study is focused on cursive handwritten Arabic numerals recognition.

In fact, a optical character recognition system can be divided into three principal steps. The first one is a pre-processing which is used for enhancing the image quality, in this context we have interested in this work to the median filter, the thresholding, the centering and the normalization techniques. The second step is the features extraction exploited to extract some efficient features called also the primitives from each image numeral that is presented in form of a matrix which will allow to convert this last to a vector which will facilitate its recognition, in order to realize this phase, we exploited the morphology method. The last phase is the recognition, in this framework we have opted the k-nearest neighbors [1-8] and the multi-layer perceptron [14-20].

Otherwise, this paper is organized as follows: In first section the proposed system is given. In second section the preprocessing process is presented. The features extraction phase is described in third section. The fourth section explains the recognition phase. The experimental results are given in fifth section. Finally, this work is ended by a conclusion.

\section{PROPOSED RECOGNITION SYSTEM} Our recognition system is presented in the following figure: 


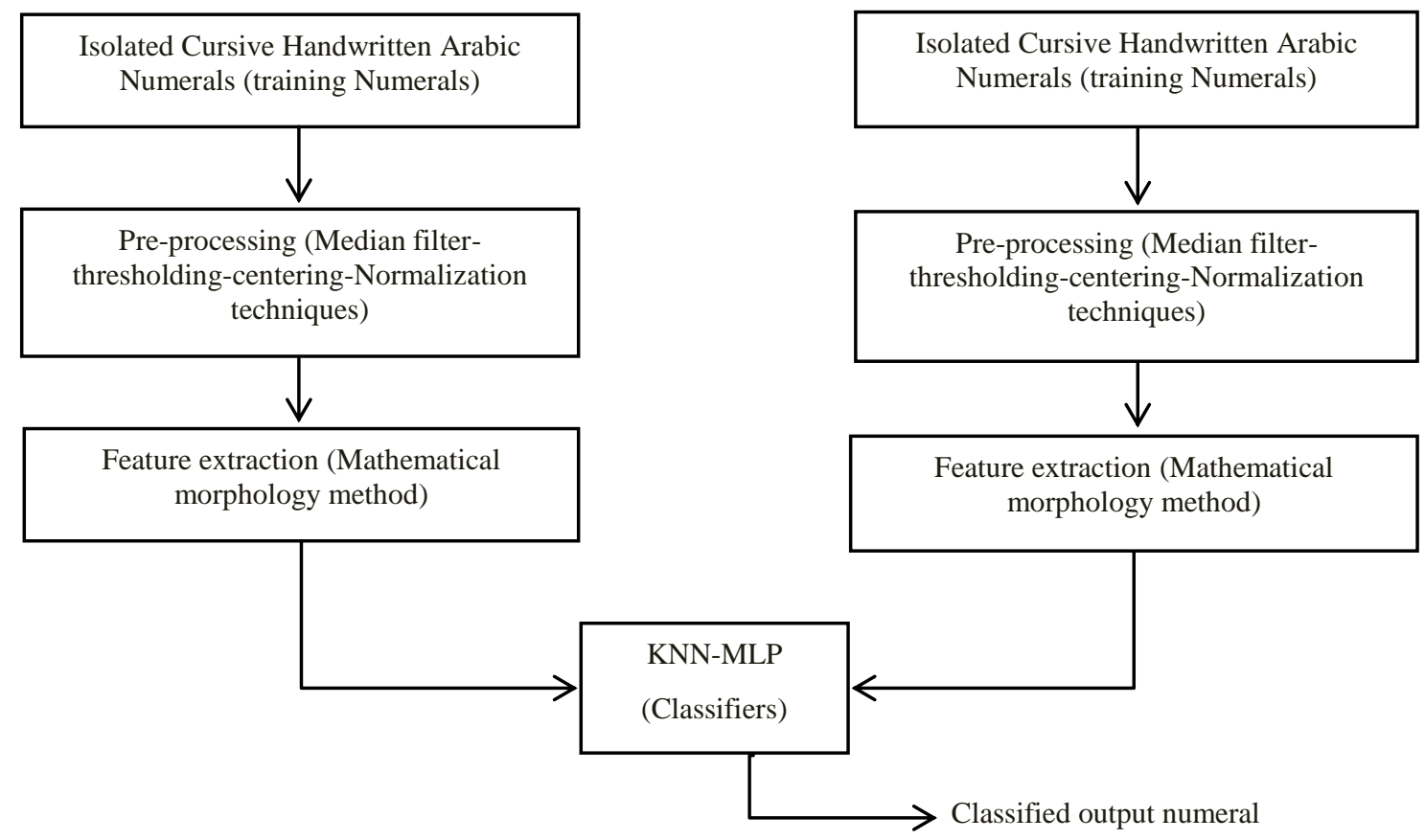

Fig 1: The proposed system for cursive handwritten Arabic numerals recognition.

\section{CURSIVE HANDWRITTEN ARABIC NUMERALS DATABASES}

The learning database that we have used contains 7000 handwritten Arabic numerals ranging from 0 to 9. Which are
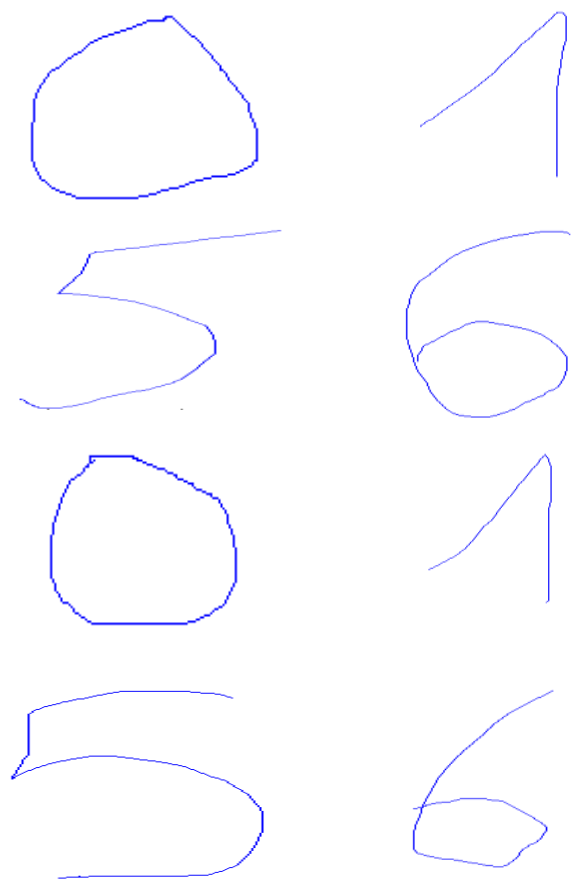
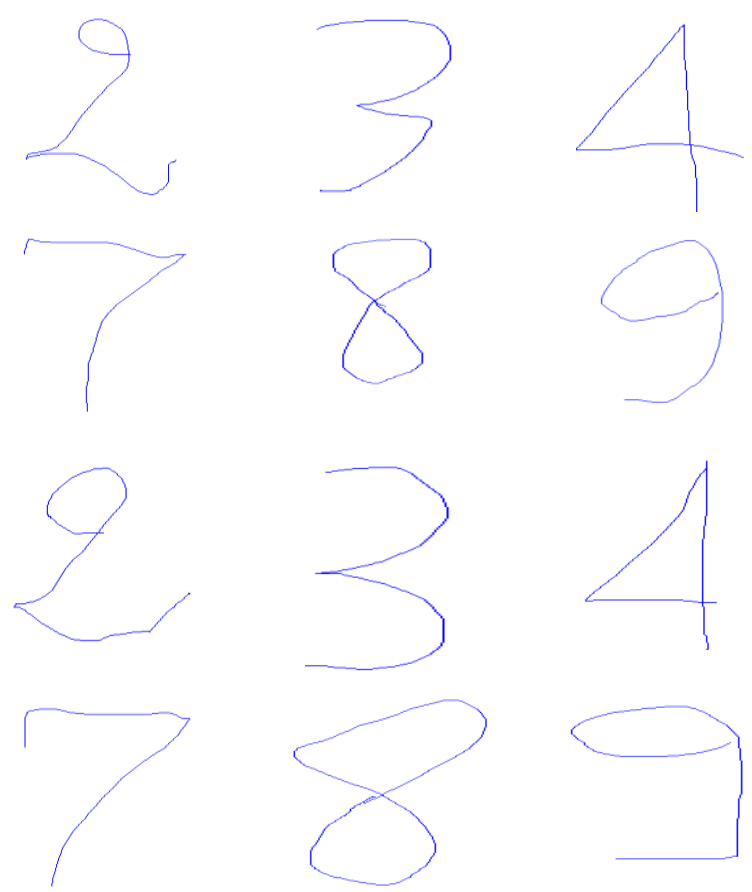

Fig 2: Example of cursive handwritten Arabic numerals written by several different scripters.

\section{PRE-PROCESSING}

Pre-processing for image is concentrated on noise removal and details-enhancement ant to reduce the redundant and unneeded information which will allow to produce a cleaned up version of the character image so that it can be used efficiently by the feature extraction components of the OCR. In this work, we have pre-processed each image numeral by a median filter exploited for removing each noise from image and thresholding used in order to render each image 
containing only the black and white colors according a preset threshold then we used the centering technique so that the numeral can be positioned just in center of its image, finally we have used the normalization technique in order that to normalize all sizes of numeral image.

- Median filter :

- Thresholding technique:

- Centering technique :

- Normalization technique :

\section{FEATURES EXTRACTION}

Feature Extraction is the operation by which certain features of interest within an image are extracted and detected and represented for further processing. The term 'feature' refers to similar characteristics. Therefore, the main goal of a features extraction phase is to accurately retrieve these features. In this context many methods can be used to compute the features. In this study, we use the mathematical morphology [9-13] method which is can be explained as follows:

In fact, the features extraction is based on mathematical morphology .The characteristic areas can be detected by the dilatation of the numeral image processed in four directions.

The characteristic zones can be detected by the intersections of dilations found to the East, West, North and South.

Each point belongs to the characteristic area if and only if:

- This point does not belong to the limit of the object.

- From this point, moving in a straight line to the South, North, East and West we cross the object. The result of the extraction is illustrated in the (Figure 4).

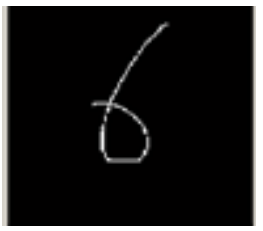

Fig 3: Image of numeral 6 before the mathematical morphology method.

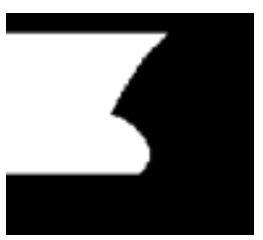

Fig 4: Features extraction by mathematical morphology method from numeral 6 (East dilatation).

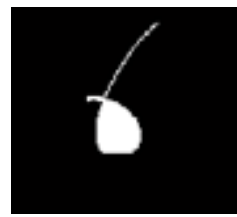

Fig 5: Features extraction by mathematical morphology method from numeral 6 (Central zone).

\section{LEARNING-CLASSIFICATION PHASE 6.1 The $K$ nearest neighbors}

$\mathrm{K}-\mathrm{Nearest-Neighbors}(\mathrm{KNN})$ [1-8] classifier is an efficient method for classification problems without any need of learning. It works by calculating the distances between a unknown vector (numeral of test) and a set of vectors (numerals of learning base) whose each of them its class is known. A k-nearest-neighbors classifier takes into account only the $\mathrm{k}$ nearest prototypes to the unknown numeral, and the majority of class values of the $\mathrm{k}$ neighbors determine the decision. In the k-nearest neighbors classification, the unknown numeral is assigned to the class which is most represented, for example the figure 6 shows that the class of unknown vector is the class 1 . In this context, it must be noted that the importance of choosing of the number $\mathrm{k}$ is indisputable.

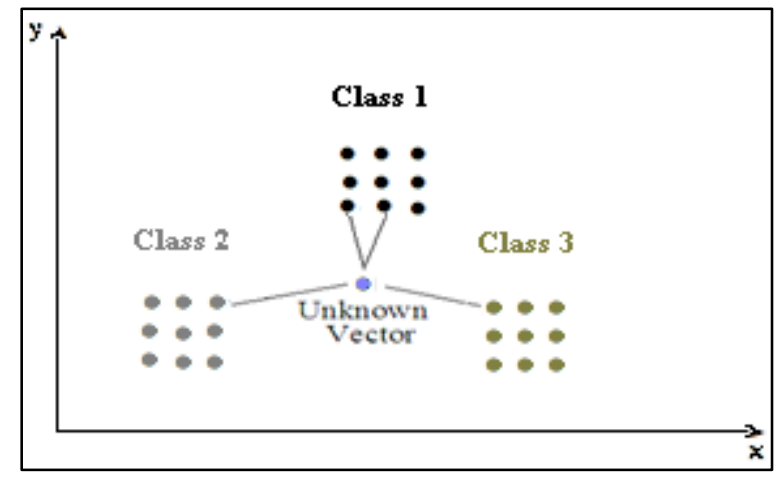

Fig 6: 4-NN of unknown vector from 3 different classes.

\subsection{The neural network multi-layer perceptron}

The Neural Network [14-20] presented in this figure is a multi-layer perceptron that we have used in our work.

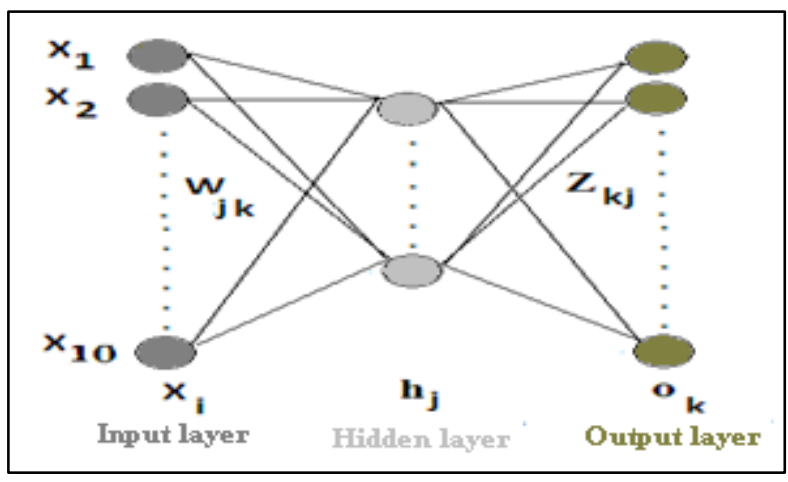

Fig 7: The multi-layer perceptron (MLP).

A perceptron multi-layer is widely used as a classifier in many character recognition systems. Also, due to the simplicity, generality, and good learning ability of neural networks, it contains the following elements:

- An input layer of $\mathrm{N}$ vectors whose each of them represents a numeral, each vector has $\mathrm{M}$ components (features vector : $\mathrm{X}_{\mathrm{i}}$ ).

- A hidden layer of $\mathrm{P}$ activations neural $\mathrm{h}_{\mathrm{j}}$.

- An output layer of $\mathrm{N}$ activations neural $\mathrm{o}_{\mathrm{k}}$.

- $\mathrm{N} \times \mathrm{P}$ connections between input layer and hidden layer, each weighted by $\mathrm{W}_{\mathrm{jk}}$. 
- P×N connections between hidden layer and output layers, each weighted by $Z_{k j}$

The learning of perceptron multi-layer is realized by the back propagation algorithm in five steps which are:

Step I. Initialize the network, with all weights $\mathrm{W}$ and $\mathrm{Z}$ set to random numbers between -1 and +1 .

Step II. Present firstly the vectors $\mathrm{X}$ whose all numerals are converted numerals and obtain the real output which is calculated as a image value of a dot matrix produce by a logistic function $\mathrm{f}(\mathrm{x})=\frac{1}{1+e^{-x}}$

Step III. Compare the real output with the desired output which is a 10-element vector with a 1 in the position of the numeral it represents, and 0's everywhere else. For example, the numeral " 0 " is to be represented by a 1 in the first element (as "0" is the first numeral of the Arabic numerals), and 0's in elements two through 9 .

Step IV. Propagate the error backward.

(a) Correct the weights of output layer Z.

(b) Correct the input weights $\mathrm{W}$.

Step V. Take the average difference between the desired and the output vector to calculate the error.

Step VI. Repeat from Step II until the real output converges to the desired output.
After the learning of MLP, for classifying the test numeral, using the Euclidean distance between the vector whose this characteris converted by the mathematical morphology and each of the desired output (vector ok, $\mathrm{k}=0,1, \ldots, 9$ ).

Nextly, the recognition will be attributed to the numeral that is very nearest to test numeral.

\section{EXPERIMENTAL RESULTS}

As we have already cited, the intended purpose of this study is to compare the performance of two methods of learningclassification which are the multilayer perceptron and $\mathrm{K}$ nearest neighbors by using during the features extraction phase the mathematical morphology.

Furthermore, we propose to know the variation impact of hidden layer neurons number on the performances of MLP classifier in one hand, and the effect variation of number of $\mathrm{k}$ nearest neighbors on the performances of the k-NN classifier in other hand. Saying the performances, this means the recognition rate of each numeral $\tau_{\mathrm{n}}$ as well as that global that is to say of all the numerals.

To do this, we have chosen the values $\{1,10,20\}$ of hidden layer neurons number. And the values $\{6,10,15\}$ of number of $\mathrm{k}$ nearest neighbors.

After having implemented these two recognition systems we obtained the results shown in the following table:

Table 1. The recognition rate of each cursive handwritten Arabic numeral $\tau_{\mathrm{n}}$ and that global $\tau_{\mathrm{g}}$ which are given in $\%$.

\begin{tabular}{|c|c|c|c|c|c|c|}
\hline \multirow{3}{*}{ numerals } & & \multirow{2}{*}{\multicolumn{2}{|c|}{$\begin{array}{c}\text { MLP } \\
\text { layer neurons number }\end{array}$}} & \multirow{2}{*}{\multicolumn{3}{|c|}{$\begin{array}{c}\text { KNN } \\
\text { Number of k nearest neighbors }\end{array}$}} \\
\hline & Hidden layer neurons number & & & & & \\
\hline & $\begin{array}{c}\tau_{n} \\
1 \text { neuron }\end{array}$ & $\begin{array}{c}\tau_{\mathrm{n}} \\
10 \text { neurons }\end{array}$ & $\underset{\tau_{n}}{20 \text { neurons }}$ & $\begin{array}{c}\tau_{n} \\
K=6\end{array}$ & $\begin{array}{c}\tau_{\mathrm{n}} \\
\mathrm{K}=10\end{array}$ & $\begin{array}{c}\tau_{\mathrm{n}} \\
\mathrm{K}=15\end{array}$ \\
\hline $\mathbf{0}$ & 78.00 & 100.00 & 100.00 & 89.00 & 91.00 & 99.00 \\
\hline 1 & 87.00 & 97.00 & 97.00 & 91.00 & 92.00 & 96.00 \\
\hline 2 & 20.00 & 89.00 & 95.00 & 82.00 & 86.00 & 92.00 \\
\hline 3 & 80.00 & 51.00 & 79.00 & 74.00 & 79.00 & 81.00 \\
\hline 4 & 96.00 & 63.00 & 94.00 & 82.00 & 86.00 & 86.00 \\
\hline 5 & 40.00 & 93.00 & 93.00 & 77.00 & 79.00 & 89.00 \\
\hline 6 & 47.00 & 92.00 & 94.00 & 65.00 & 70.00 & 76.00 \\
\hline 7 & 82.00 & 82.00 & 98.00 & 55.00 & 64.00 & 74.00 \\
\hline 8 & 35.00 & 45.00 & 52.00 & 64.00 & 75.00 & 73.00 \\
\hline 9 & 46.00 & 85.00 & 91.00 & 58.00 & 61.00 & 78.00 \\
\hline$\tau_{\mathrm{g}}$ & 57.22 & 79.70 & 89.20 & 73.70 & 78.30 & 84.40 \\
\hline
\end{tabular}

The associated graph to table above is: 


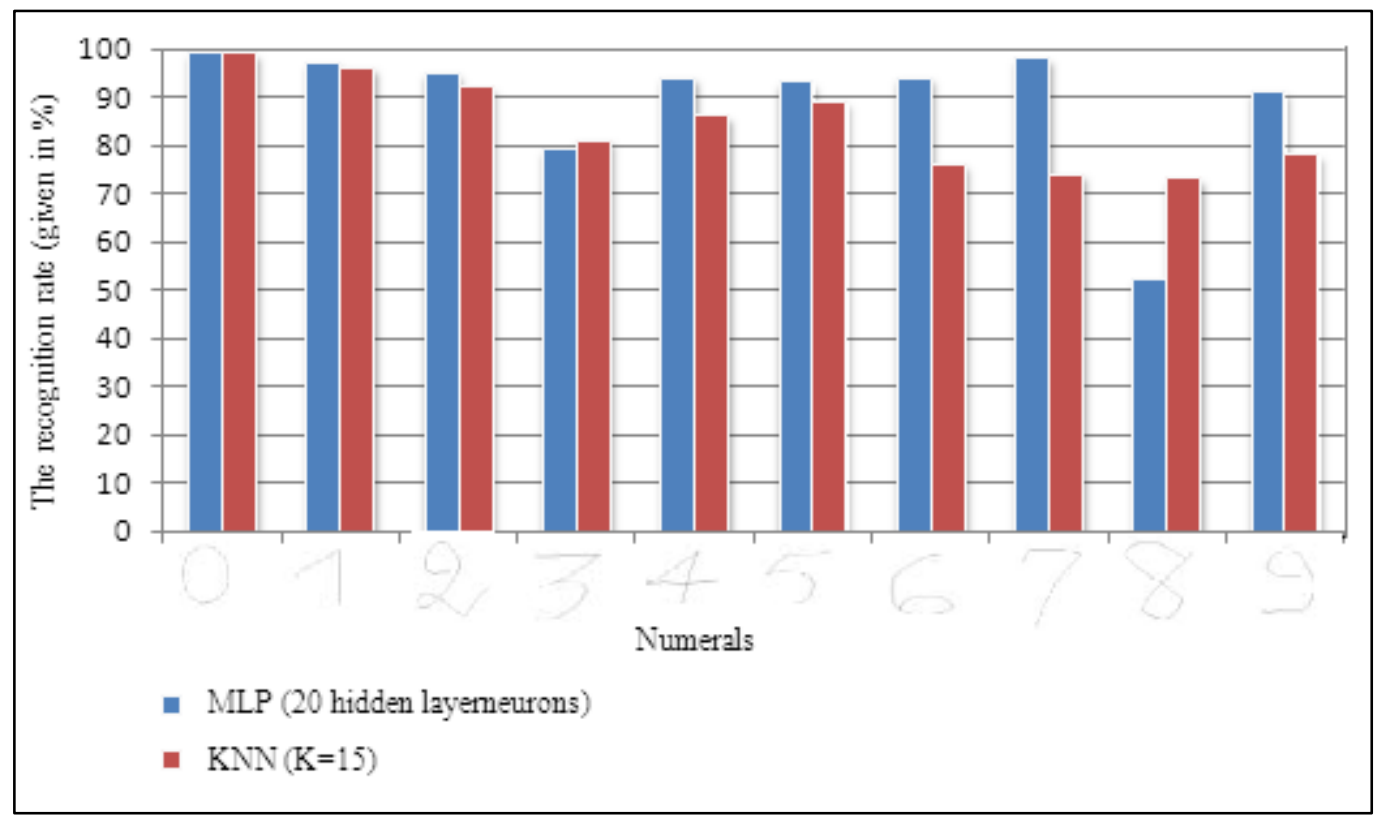

Fig 8: The graph of recognition rate of each cursive handwritten Arabic numeral $\tau_{\mathrm{n}}$ by using the MLP (20 hidden layer neurons) and the KNN (K=15)

\section{- Analysis and comment}

Taking into account the results obtained after having implemented this recognition systems, we can effectively conclude that the MLP which is most performer is that when the number of hidden layer neurons is equal to 20 in first time and the $\mathrm{KNN}$ which is the most efficient is that when $\mathrm{K}=15$ in second time. Furthermore, the MLP is more powerful than the $\mathrm{KNN}$ in cursive handwritten Arabic numerals. Moreover, in order to improve this work we have introduced the following graphical interface that includes all steps of the recognition systems that we used:

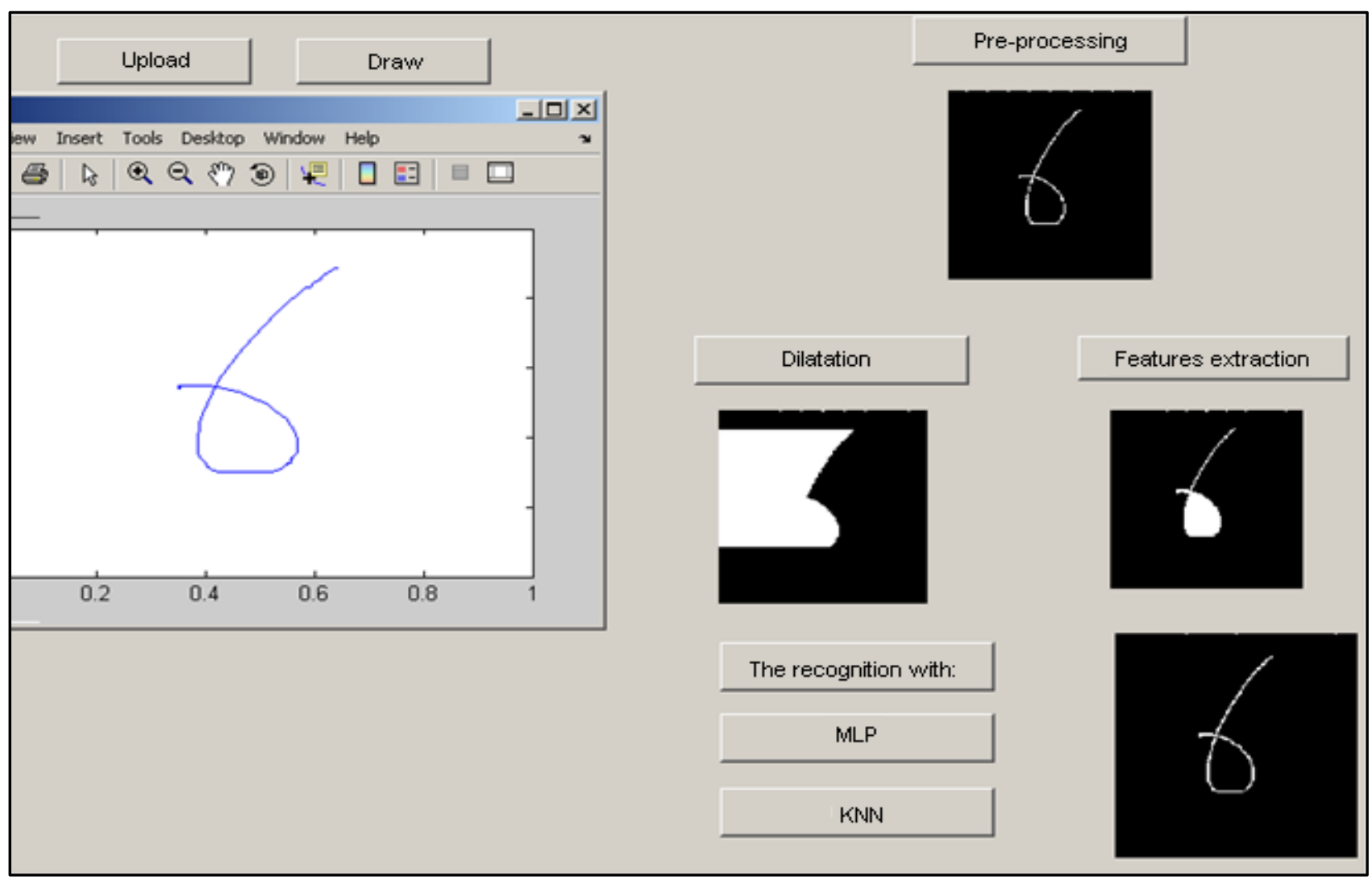

Fig 9: Elaborated graphical interface for cursive handwritten numerals recognition using both classifiers MLP and KNN. 


\section{CONCLUSION}

In this paper, an experimental evaluation on cursive handwritten Arabic numerals recognition was introduced by using in the preprocessing phase the median filter, the thresholding, the centering and the normalization techniques, in the features extraction we use the mathematical morphology. Finaly, in the recognition phase we use the knearest neighbors and the multi-layer perceptron. The achieved results demonstrates that the performances are increased if the number of hidden layer neurons is also increased when using the MLP classifier and if the number of nearest neighbor is also increased when using the KNN classifier in one hand. And that the MLP is more performing than the KNN in this recognition in other hand. In perspective, to increase the system reliability will it be possible to use the new hybridizations at the level of the features extraction such as the mathematical morphology and the zoning or the mathematical morphology and the zig-zag. Finaly, we can extend the application of this recognition system for the others characters.

\section{REFERENCES}

[1] L. Li and L. Zhang, J. SU. Handwritten character recognition via direction sring and nearest neighbor matching. The Journal of China Universities of Posts and Telecommunications, Volume 19, Supplement 2, October 2012, Pages 160-165,196.

[2] Z. Liu, Q. Pan, J. Dezert. A new belief-based K-nearest neighbor classification. Pattern Recognition, Volume 46, Issue 3, March 2013, Pages 834-844.

[3] S. H. Rodríguez, J. F. M. Trinidad, J. Ariel C. Ochoa. Fast $\mathrm{k}$ most similar neighbor classifier for mixed data (tree k-MSN). Pattern Recognition, Volume 43, Issue 3, March 2010, Pages 873-886.

[4] N. A. Samsudin and A. P. Bradley Nearest neighbour group-based classification. Pattern Recognition, Volume 43, Issue 10, October 2010, Pages 3458-3467.

[5] T. Wakahara and Y. Yamashita. K-NN classification of handwritten characters via accelerated GAT correlation. Pattern Recognition, Volume 47, Issue 3, March 2014, Pages 994-1001.

[6] J.Yang and D. Zhang. From classifiers to discriminators : A nearest neighbor rule induced discriminant analysis. Pattern Recognition, Volume 44, Issue 7, July 2011, Pages 1387-1402.

[7] J. Yang, L. Zhang, J. Yang, and D. Zhang. From classifiers to discriminators: A nearest neighbor rule induced discriminat analysis. Pattern Recognition, Volume 44, Issue 7, July 2011, Pages 1387-1402.
[8] Y. LeCun, (1998). THE MNIST handwritten digit database. R.Muralidharan1, C. Chandrasekar: Object Recognition using SVM-KNN based on Geometric Moment Invariant, International Journal of Computer Trends and Technology- July to Aug Issue 2011, ISSN: 2231-2803, Page 215-220.

[9] J. Angulo and J. Serra. Automatic analysis of DNA microray images using mathematical morphology. Bioinformatics, vol 19, no 5, pp. 553-562, Mar 2003.

[10] B. El kessab, C. Daoui, B. Bouikhalene, M. Fakir, and K.Moro. Extraction Method of Handwritten Digit Recognition Tested on the MNIST Database, International Journal of Advanced Science and Technology Vol. 50, January, 2013.

[11] B. El kessab, C. Daoui, B. Bouikhalene, M. Fakir, and K.Moro. Handwritten Tifinagh Text Recognition using Neural Networks and Hidden Markov Models, International Journal of Computer Applications (0975 8887) Volume 75- No.18, August 2013.

[12] M. Iwanowski and M. Swierez. Pattern Recognition Using Morphogical Class Distrubition Functions and Classification Trees. Springer, pp. 143-154, 2011.

[13] J. Serra. Image Analysis and Mathematical Morphology. II: Theoretical Advances. Academic Press. London, 1988.

[14] S. Alma'adeed. Recognition of Off-Line Handwritten Arabic Words Using Neural Network, proc. of the Geometric Modeling and Imaging - New Trends, 2006.

[15] A. A. Desai. Gujarati handwritten numeral optical character reorganization through neural network. Pattern Recognition 43 (2010) 2582-2589.

[16] L. M. Fu Analysis of the dimensionality of neural networks for pattern recognition. Pattern Recognition, Volume 23, Issue10, 1990, Pages 1131-1140.

[17] K. Fukushima. Recognition of partly occluded patterns: A neural network model. Biol. Cyber net. vol. 84, (2001), pp. 251-259.

[18] P. Melin Modular Neural Networks and Type-2 Fuzzy Systems for Pattern Recognition. Springer Volume 389 2012

[19] P. Nagare. License Plate Character Recognition System using Neural Network. International Journal of Computer Applications, Volume 25, No. 10, July 2011, pp. 36-39.

[20] I.S. Oh and C.Y. Suen. A class-modular feed-forward neural network for handwriting recognition, Pattern Recognition, 35: 229-244, 2002. 